\title{
Superficial structures cartography of the Essaouira basin under ground (Morocco), by small refraction seismic: contribution of the static corrections in the reinterpretation of the speeds variations.
}

\author{
M. DAHAOUI ${ }^{1}$, L. AIT BRAHIM ${ }^{3}$, A. El ADRAOUI ${ }^{2}$, A. ABDELOUAFI ${ }^{6}$, M. DAKKI $^{4}$ and A. El IMRANI ${ }^{5}$ \\ ${ }^{1,3}$ Université Mohammed V, Faculté des Sciences, laboratoire Géophysique et Risques naturels, structure Géorisk, \\ Avenue Ibn Battouta, BP 1014, Rabat, Maroc \\ ${ }^{2,6}$ Université Mohammed V, Faculté des Sciences, laboratoire GEOS, structure GEORISK, Avenue Ibn Battouta, \\ BP 1014, Rabat, Maroc \\ ${ }^{4,5}$ Office National d'Hydrocarbures et des Mines, (ONHYM), Rabat, Maroc
}

\begin{abstract}
The static corrections are a necessary step in the sequence of the seismic processing. This paper presents a study of these corrections in the Essaouira basin. The main objective of this study is to calculate the static corrections by exploiting the seismic data acquired in the field to improve the deep structures imaging. It is to determine the roof and the basis of the superficial layers which constitute the weathered zone while calculating the delays of seismic wave's arrivals in these layers. The purpose is to cancel the effect of the topography and the weathered zone, in order to avoid any confusion when the seismic and geological interpretation. The results obtained show the average values of the static corrections varying between - 127 and $282 \mathrm{~ms}$ (double time), with existence of high values by location, particularly in the Eastern and North-Eastern of the basin, which meant the presence of altered zone with irregular topography and whose thickness and speeds vary laterally. In effect the variations of velocities in the fifty meters from the surface may introduce significant anomalies in seismic refraction, with heavy consequences when the interpretation or the drilling establishment. These variations are mainly due to lateral changes in facies and variations in the formations thickness. The calculation of the static corrections, revealed high values at certain areas (East and North-East), which will enable us to better orient the future campaigns in these zones. It is therefore necessary to concentrate the seismic cores drillings and the small refraction seismic profiles by tightening the seismic lines meshes in order to have the maximum values of static corrections and thereafter a better imaging of the reflectors.
\end{abstract}

Keywords: Essaouira basin, Static corrections, seismic refraction, weathered zone, seismic waves, lateral variation of lithology.

\section{Introduction}

Les bassins marocains ont été depuis longtemps l'objet de plusieurs recherches pétrolières justifiées par l'intérêt économique que présentent certaines formations géologiques. Or ces recherches n'ont découvert que de modestes réserves dans le bassin d'Essaouira et le bassin de Rharb. Cependant plusieurs efforts ont été déployés dans l'exploration pétrolière au Maroc au cours des dernières années. Les bassins sédimentaires ont fait l'objet de plusieurs campagnes d'exploration, réalisées par différents établissements spécialisés dans le domaine [1].

La prospection pétrolière consiste à explorer le sous-sol en utilisant l'imagerie sismique, fondée sur le principe physique de transmission des ondes dans les milieux solides dans le but d'analyser les structures géologiques en profondeur, et la découverte des zones de piégeage des hydrocarbures, avant de recourir au forage, qui présente un outil extrêmement coûteux [2]. Dans ce cadre, d'importantes méthodes géophysiques sont mises en œuvre: la sismique réflexion à couverture multiple est la plus utilisée actuellement en acquisition des données de terrain. Cette méthode fournit une échographie du sous-sol en deux ou trois dimensions selon la mise en œuvre de chacune sur le terrain [8].

Les couches superficielles existantes aux dessus d'un réservoir (Zone Altérée) constituent un problème non négligeable, étant donné que la propagation des ondes sismique dans ces couches engendre un retard sur le temps d'arrivée [9].

Dans ce travail, on se concentre sur l'étude de la zone altérée (Weathered Zone : WZ) par le calcul des 
corrections statiques à appliquer aux données sismiques en utilisant deux approches différentes, à savoir : la méthode des carottages sismiques (Upholes) et celle de la sismique petite réfraction [7].

\section{Cadre géologique}

Le bassin d'Essaouira qui fait partie du bassin côtier d'El Jadida-Agadir [8], se localise entre 9०46'11" W et $31^{\circ} 30^{\prime} 44^{\prime \prime} \mathrm{N}$ et s'étend sur une superficie d'environ $20000 \mathrm{~km}^{2}$. Il est limité au Nord par le bassin de Doukkala au Nord-Est par les Jebilet occidentales, à l'Est par la plaine du Haouz, au Sud-Est par le Haut Atlas occidental, au Sud par les plateaux de Haha et à l'Ouest par l'Océan Atlantique (figure 1).

Le bassin d'Essaouira est caractérisé par des dépôts paléozoïques qui ont été largement affectés, au Carbonifère, par l'orogénèse hercynienne. En effet, durant le Trias et le début du Lias, les dépôts ont été contrôlés par des failles normales extensives entrainant la formation de blocs basculés et d'épaisses séries détritiques rouges, de sel et de dolomies. Durant le Jurassique et le Crétacé, la sédimentation a été caractérisée par des carbonates marins de plate-forme et des anhydrites qui furent interrompus par plusieurs intervalles silicoclastiques [1].

Sur presque tout l'arrière-pays affleurent les formations du Crétacé; des formations du Tertiaire (couches phosphatées) et du Quaternaire (dépôts superficiels) s'y rencontrent localement dans des cuvettes synclinales. La zone côtière est presque partout recouverte par les formations dunaires du Pliocène et du Quaternaire, sur une bande parallèle à l'Océan et large d'une vingtaine de kilomètres [4]. (Figure1).

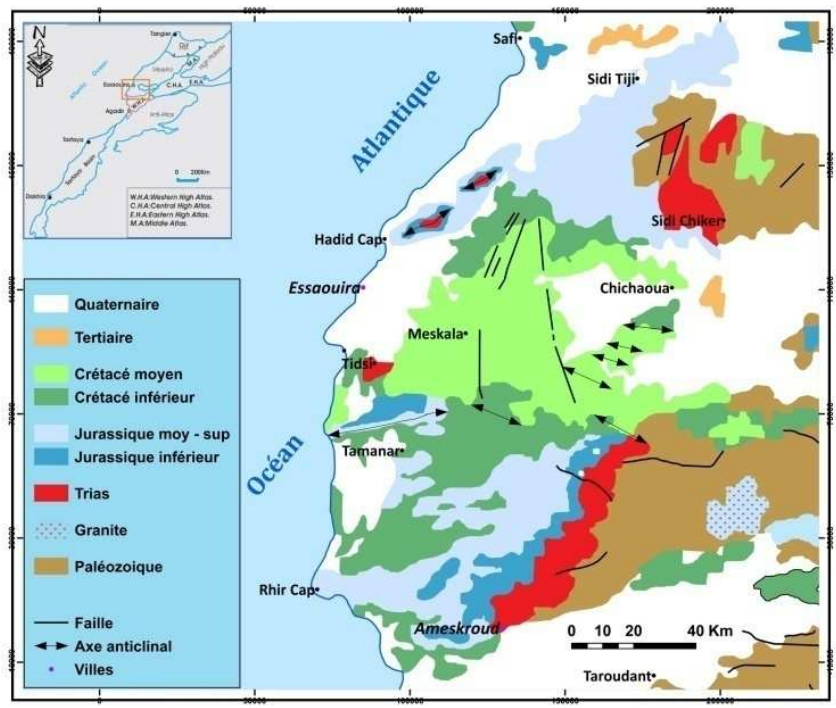

Fig. 1. Carte de localisation et contexte géologique du bassin d'Essaouira.

\section{Outils et méthodes}

Pour atteindre les objectifs fixés, nous avons utilisé deux méthodes complémentaires : 1) Nous avons commencé par l'analyse des résultats de calculs par la méthode de la petite réfraction afin d'évaluer les caractéristiques de la couche altérée, cerner l'architecture du bassin en deux dimensions, et suivre sont évolution spatio-temporelle (temps en fonction de profondeur). Cette méthode permet d'avoir l'épaisseur et la vitesse moyenne de la couche entre le tir directe et le tir inverse [3], mais pour avoir des valeurs de la correction statique entre ces couplets de points de mesures l'interpolation par les carottages sismiques (Upholes) s'avère nécessaire comme solution pour une étude complète de la zone altérée [5].

Les corrections statiques correspondent généralement à des valeurs utilisées pour compenser les retards sur le temps d'arrivée en sismique dues aux effets de l'altimétrie et de l'altération des couches superficiels. Le nom de statique provient de la constance des corrections en fonction de temps c'est-à-dire que pour une trace brute donnée, tout le train d'onde est déclaré lors de ces corrections de la même valeur de temps. Les valeurs de la correction statique peuvent être positives ou négative selon le positionnement des traces par rapport au plan de référence (Datum Plane : DP) [3, 5]. Ci-dessous la formule (1) pour calculer les corrections statiques [3, 7].

$\mathrm{CS}=\left\{\left[\sum(\mathbf{h i} / \mathbf{v i})+\left(\mathbf{E d}-\mathbf{E s}+\sum \mathrm{hi}\right)\right] / \mathrm{Vc}\right\}$

Avec: Vi et hi: sont respectivement la vitesse et l'épaisseur de la couche i déduites de la dromochronique.

Zs: Elévation de point de mesure par rapport au niveau zéro de la mer obtenue par les levées topographiques.

Ed: Elévation du plan de référence (DP) qui égale à $400 \mathrm{~m}$ dans la zone d'Essaouira.

Vc: La vitesse de remplacement estimée à partir de la connaissance préalable $(\mathrm{Vc}=3000 \mathrm{~m} / \mathrm{s}$ dans le bassin d'Essaouira).

2) Dans un deuxième temps, et dans le cadre de l'évolution de la propagation des vitesses au niveau du bassin nous avons étudié la répartition lithologique à 
partir des données de forages réels les plus représentatives (figure 4) pour estimer d'une part le taux de variation des vitesses en fonction de la variation lithologique [6], et d'autre part pour comparer l'harmonisation des résultats obtenus par cette méthode avec celle de la petite réfraction. Il nous semble obligatoire avant toute modélisation de préparer les données nécessaires de la zone d'étude. Ces dernières sont collectées à partir des études faites sur la zone altérée par la méthode de la petite réfraction et les carottages sismiques.

En utilisant des logiciels de modélisation (Petrel \& ArcGis 10.5), on a pu cartographier et modéliser les différents paramètres de la zone altérée, sous forme des cartes des iso-vitesses, d'épaisseurs (isopâques) et des carottages vitesses-lithologie pour chacune des couches mise en évidences dans cette étude, où chaque point est placé selon ses coordonnées XY.

\section{Résultats}

Bien que les points des carottages sismiques ne sont pas trop nombreux et ne couvrent pas une surface importante, il apparaît que les résultats obtenus pour cette méthode concordent dans l'ensemble avec ceux obtenus par la méthode de la petite réfraction (figure $5 \mathrm{~A}$ et $5 \mathrm{~B}$ ). D' autre part la fréquence de la présence ou de l'absence de ces couches ( $\mathrm{H} 0, \mathrm{H} 1$ et $\mathrm{H} 2)$ est aussi identique pour les deux méthodes. Seulement on note l'absence total d'épaisseur de la couche $\mathrm{H} 3$ par la méthode de la petite réfraction (PT), par contre sa présence au niveau de la méthode des carottages sismiques (UPH).
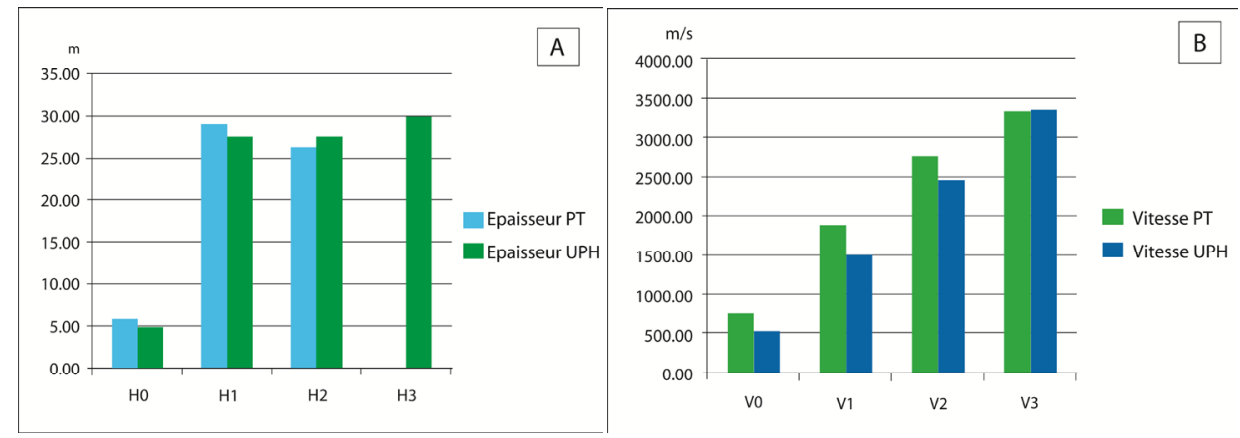

Fig. 2. A) Comparaison de la moyenne des épaisseurs des couches entre les deux méthodes. B) Comparaison de la moyenne des vitesses dans les couches entre les deux méthodes.

Les profils de corrélation montrent une variation latérale et verticale des vitesses, faisons apparaître l'existence d'une hétérogénéité lithologique du site étudié. En effet, les carottages sismiques ont permis d'identifier plusieurs formations lithologiques avec une dominance remarquable des calcaires et des argiles une fois et des calcaires et marnes une autre fois. Les deux premières couches sont généralement de même lithologie mais, parfois, la limite entre ces deux correspond à une variation lithologique.

Les résultats obtenus concernant le calcule des corrections statiques, montrent des valeurs moyennes varient entre -127 et $+282 \mathrm{~ms}$ avec l'existence des valeurs élevées par endroit notamment dans la partie Nord-Est du bassin (Figure 3).

La présente carte (figure 3) a pour objectif d'indiquer les zones où les ondes sismiques sont affectées par le maximum de retard sur les temps d'arrivée. Ces zones sont caractérisées par des valeurs de corrections statiques (Cs) importantes, qui peuvent êtres positives ou négatives. Pour remédier à ce problème, on prévoit pour la prochaine campagne sismique, de concentrer les carottages sismiques et les petites réfractions en resserrant les mailles des lignes sismiques au niveau de ces zones afin d'y avoir le maximum de valeurs de la correction statique, ce qui sera très utile afin de fournir des images assez correctes de la géométrie des réflecteurs.

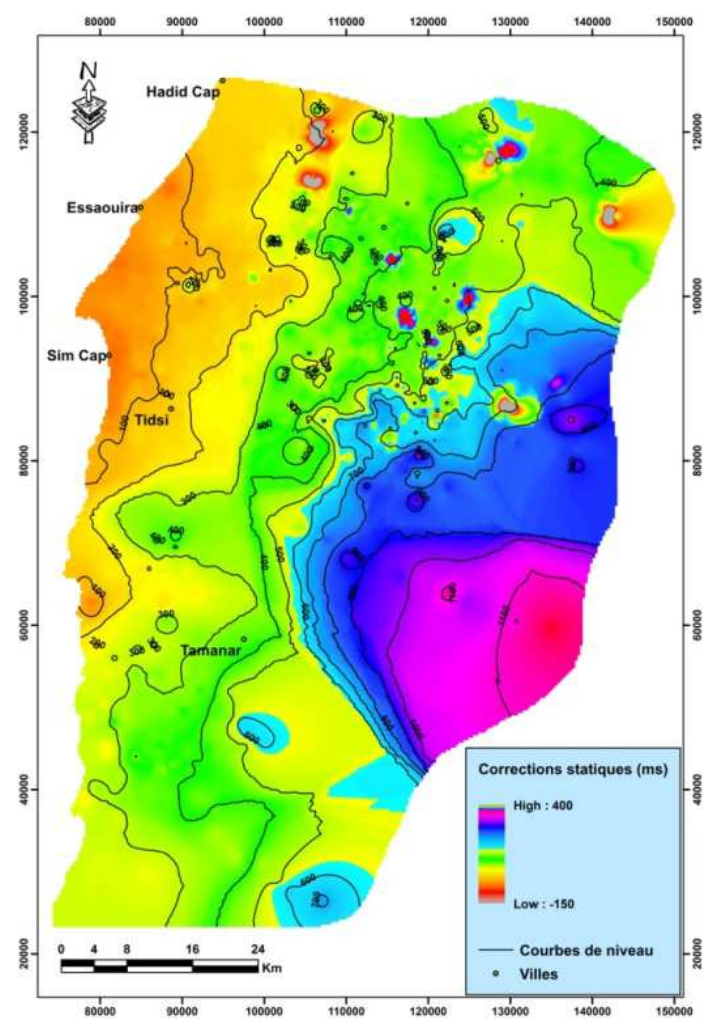

Fig. 3. Carte des corrections statiques "Cs" du bassin d'Essaouira. 


\section{Discussion \& Interprétation}

\subsection{Couches altérées (H0, H1, H2)}

Les cartes des iso-pâques de l'ensemble des couches (H0, $\mathrm{H} 1$ et $\mathrm{H} 2$ ), montrent que l'épaisseur varie latéralement dans la majeure partie des cartes. Toutefois, on remarque quelques zones très réduites et ponctuelles, indiquent une transition des couches altérées et hétérogènes vers autres qui ont des épaisseurs plus ou moins stables et homogènes (figure 4.C). La présence d'épaisseur dominant relativement la majeure partie de la carte, peut être expliqué par l'abondance de données dans ces zones par rapport aux autres, où il y a moins de données. Il est à noter que dans l'ensemble, les épaisseurs de la deuxième couche (Figure 4. B) sont plus élevées que celles de la première (Figure 4. A) (h2>h1).
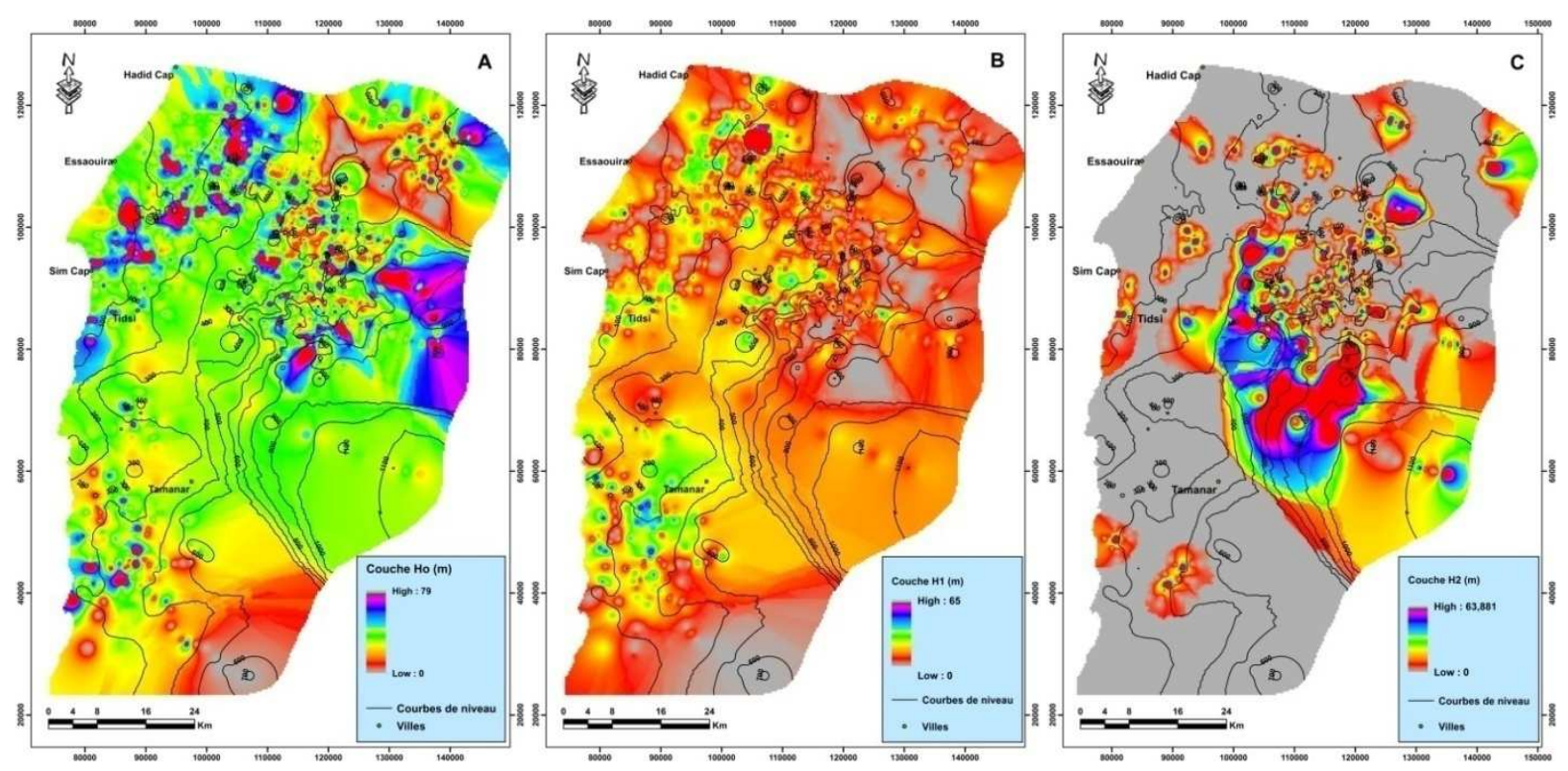

Fig. 4. A. Carte des iso-pâques de la première couche altérée « $\mathrm{H} 0$ ». B. Carte des iso-pâques de la deuxième couche altérée « H1 ». C. Carte des iso-pâques de la troisième couche altérée « $\mathrm{H} 2$ ».

En effet dans les zones de base altitude l'érosion implique une désagrégation superficielle de la roche ou du sol appelée météorisation. Cette érosion se produit sur place, et produit des débris qui restent sur place. A ces débris s'ajoutent les matériaux issus de l'érosion des zones de haute altitude. Ceci explique les épaisseurs élevées de la couche altérée dans les zones de basses altitudes. Contrairement, dans les zones de haute altitude, on distingue généralement destruction du matériel rocheux par érosion (ablation du matériel) puis son transport et son accumulation dans les zones de plus basse altitude. De ce fait, l'épaisseur de la zone altérée à hautes altitudes est relativement faible.

La présence des domaines d'épaisseurs bien concentrés au niveau de la carte, figure 4.C par rapport aux autres (figure 4.A et 4.B), indiquent une transition des couches altérées et hétérogènes vers autres qui ont des épaisseurs plus ou moins stables et homogènes. On peut dire que ces couches sont probablement liées à une zone en dehors de la zone altérée.

La variation de la somme des épaisseurs des trois couches ne montre pas une corrélation visible avec la topographie (épaisseur élevée dans les basses altitudes et épaisseur faible dans les hautes altitudes). L'explication la plus logique est que les reliefs montagneux escarpés sont des zones de dénudations, alors que les plaines et les piémonts sont des zones d'accumulation.

\subsection{Variation des Vitesses (V0, V1, V2 et V3)}

Les cartes des iso-vitesses, ressortent qu'il existe trois niveaux V0, V1 et V2, ceci indique qu'on est en présence de trois couches superficielles ayant respectivement les épaisseurs H0, H1 et H2. Dans l'analyse de quelques dromochroniques, on peut rencontrer une quatrième vitesse V3 d'une épaisseur non déterminée.

$\mathrm{Au}$ niveau de la première couche, les vitesses (Figure 5. A) sont largement inférieures à celles de la deuxième (figure 5.B) (V1>V0). Cette différence correspond probablement, soit à une différence de lithologie entre la couche $\mathrm{H} 0$ et $\mathrm{H} 1$, soit aux roches de même lithologie mais peu altérées en $\mathrm{H} 1$ par rapport à H0. Etant donné que la vitesse de remplacement de la zone d'Essaouira est fixée à $3000 \mathrm{~m} / \mathrm{s}$, on peut considérer que les vitesses relativement élevées de la troisième couche (figure 5.C), correspondent à celles des roches dures non altérées, de même pour la quatrième couche (figure 6) qu'on peut la considérée en de hors de la zone altérée. La distribution de la vitesse dans ce dernier cas montre une assez bonne corrélation avec la topographie, ou les basses altitudes correspondent aux zones de faibles vitesses et les hautes altitudes correspondent aux zones de fortes vitesses. 


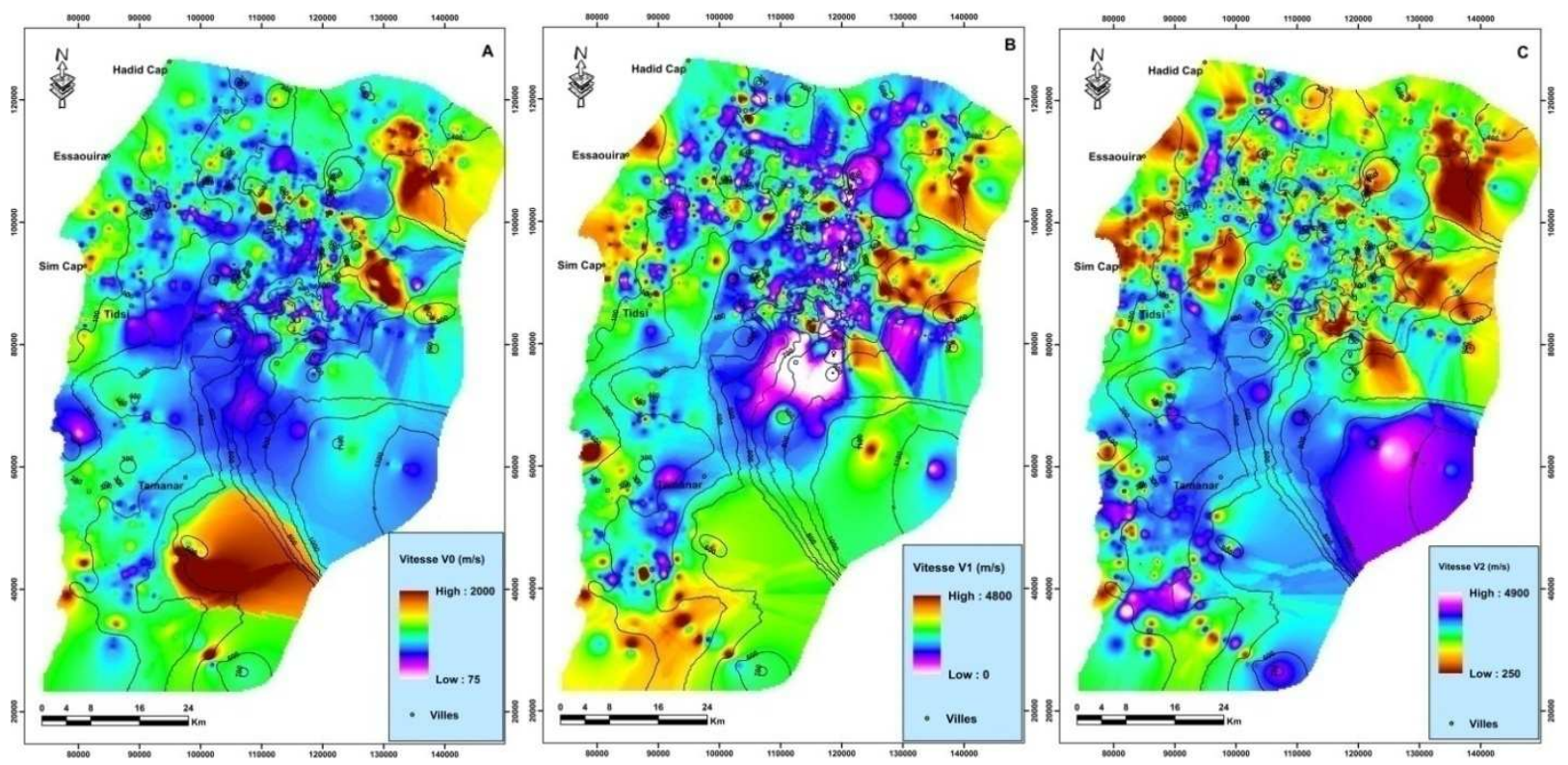

Fig. 5. A. Carte des iso-vitesses de la première couche "H0". B. Carte des iso-vitesses de la deuxième couche "H1". C. Carte des isovitesses de la troisième couche "H2".

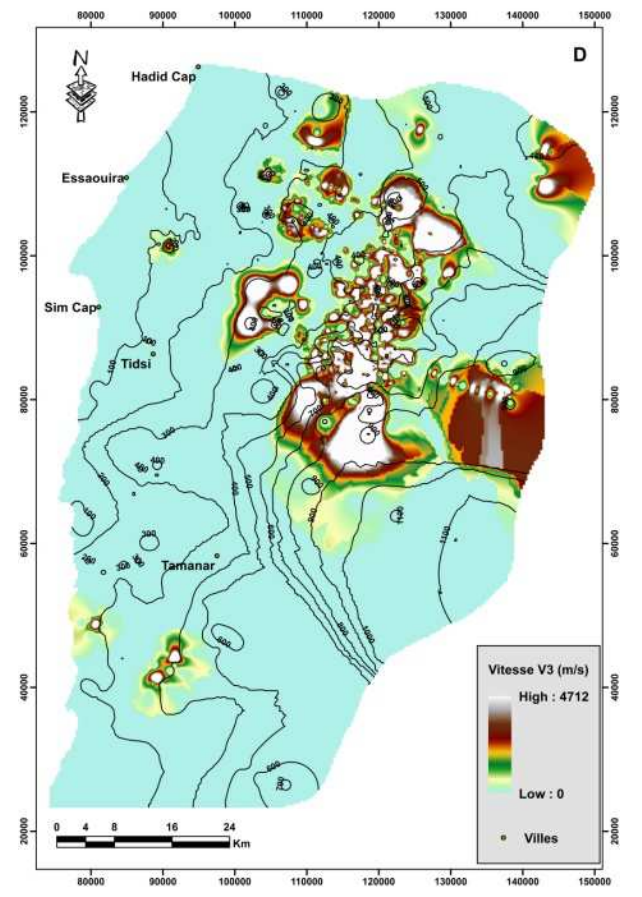

Fig. 6. Carte des iso-vitesses de la quatrième couche "H3".

D'après les trois vitesses enregistrées, il apparaît que $\mathrm{V} 0<\mathrm{V} 1<\mathrm{V} 2$. Donc, la vitesse augmente avec la profondeur car les roches deviennent plus consolidées et très peu altérées. On peut obtenir aussi sur ce site que le recouvrement du substratum représente un profil complexe et hétérogène (altéré).

\subsection{Corrélation vitesses-lithologies}

Les méthodes de calcules présentées précédemment, nous ont permis de suivre la variation des épaisseurs des différentes couches de la zone altérée en se basant sur la variation des vitesses des ondes sismiques qui $y$ propagent. D'autre part, les valeurs des vitesses peuvent être corrélées avec la lithologie probable et la lithologie réelle obtenus par les carottages.

L'analyse des carottages obtenus ont permis d'identifier plusieurs formations lithologiques, ces formations, nous ont permis de réaliser deux coupes de différentes directions (NS, EW) afin de montrer la relation entre la vitesse sismique et la variation lithologique.

Le profile Nord-Sud (Figure 7.A) montre une élévation progressive du terrain vers le Sud, ainsi la lithologie de la zone altérée est variable verticalement et discontinues latéralement avec des passages latéraux fréquents, ceci peut être expliqué par la grande distance séparant les carottages (2 à $5 \mathrm{Km}$ ). Le profil de corrélation montre que les vitesses varient latéralement et verticalement, ainsi apparaît l'existence d'une hétérogénéité lithologique du site. Par endroits, les terrains où les vitesses sont inférieures à $700 \mathrm{~m} / \mathrm{s}$ correspondent à des argiles, des marnes ou des calcaires qui sont très altérés (puits UH41, UH19). Par contre les vitesses voisinent les $3000 \mathrm{~m} / \mathrm{s}$, sont des marnes et des calcaires très tendres. Ces vitesses sont celles des roches non altérées.

Les deux puits situant à l'Est de la coupe orientée EstOuest (U20 et U21) (Figure 7.B), sont constitués essentiellement par des calcaires avec toutes ses variétés et c'est au niveau de ces puits où les vitesses atteignent son maximum : 2500 et $2700 \mathrm{~m} / \mathrm{s}$ vers une profondeur de $470 \mathrm{~m}$. Au niveau de cette coupe (figure 7.B), la valeur la plus élevée $(2700 \mathrm{~m} / \mathrm{s})$, se présente au niveau de la base de la couche altérée du puits UH21, ceci est dû à une alternance des couches où la lithologie est dominée par les calcaires et parfois avec des marnes. Cependant la valeur minimale est de l'ordre de $440 \mathrm{~m} / \mathrm{s}$, apparaît au sein de la première couche altérée dans les formations du calcaire et du marne au niveau du puits UH29, ceci indique l'influence de la densité sur la vitesse de propagation des ondes sismiques. 

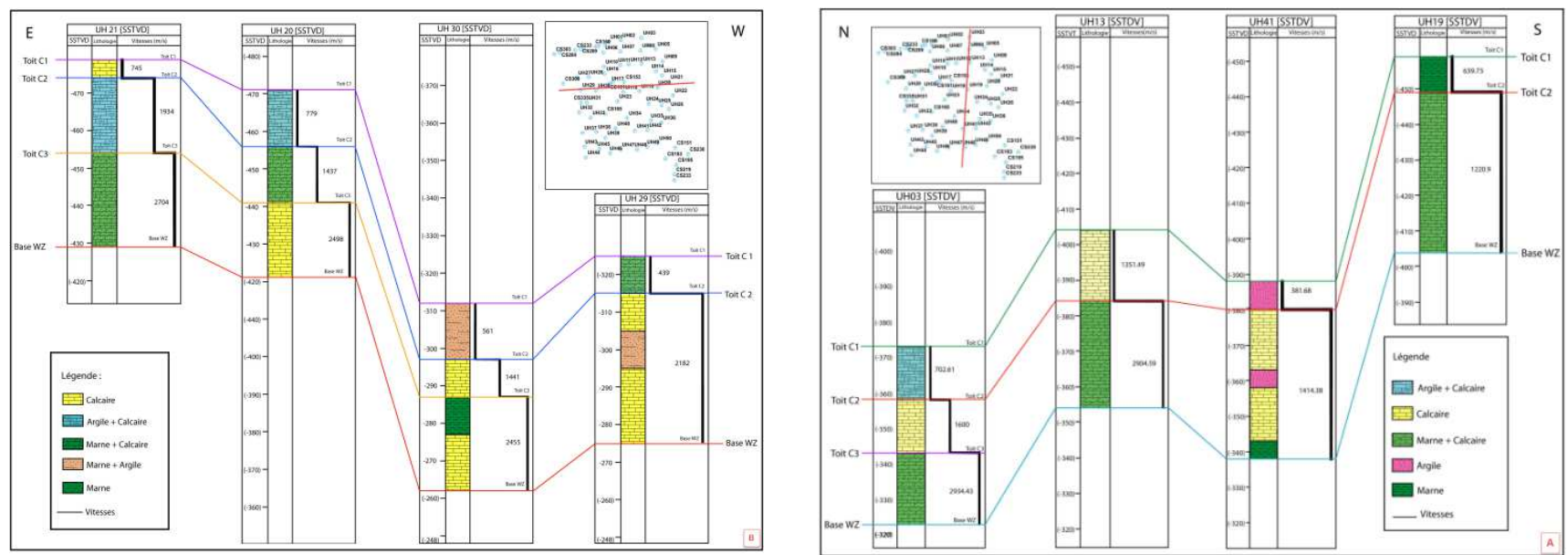

Fig. 7. A. Profil de corrélation 'vitesses-lithologie' de la zone altérée (transect Nord-Sud). B. Profil de corrélation 'vitesses-lithologie' de la zone altérée (transect Est-Ouest).

\section{Conclusion}

La sismique réflexion associée à la petite réfraction est un outil d'investigation extrêmement puissant joignant une grande profondeur de pénétration à une bonne résolution. Son coût est relativement élevé par rapport aux autres méthodes géophysiques, mais il demeure très compétitif par la précision de son diagnostic et la finesse de ses informations.

L'analyse détaillée des résultats de calcul des corrections statiques a dégagé la présence d'une zone altérée de topographie irrégulière dont l'épaisseur et les vitesses varient latéralement. Ces facteurs conduisent à souligner l'importance et la minutie à l'application des corrections statiques au cours $\mathrm{du}$ traitement des données sismiques issues du secteur d'étude. En effet les variations de vitesses dans les cinquante premiers mètres peuvent introduire des anomalies importantes sur la sismique (entre -127 et $282 \mathrm{~ms}$ : temps double de retard) pouvant avoir de lourds conséquences lors de l'interprétation ou de l'implantation de forage. Ces variations sont principalement dues à des changements latéraux de faciès et des variations d'épaisseur des formations.

Le calcul des corrections statiques obtenues par les deux méthodes, a mis en évidence des valeurs élevées au niveau de certaines zones (Nord-Est), ce qui nous ramène à faire un certain nombre de recommandations qui permettront d'orienter les futures campagnes au niveau de ces zones. A savoir d'y concentrer les carottages sismiques et les petites réfractions, en resserrant la mailles des lignes sismiques, afin d'y avoir le maximum de valeurs de la correction statique et par la suite une meilleur imagerie des réflecteurs.

\section{Remerciement}

Nous remercions l'ONHYM (DEP) pour avoir mis à notre disposition les enregistrements et les carottages sismiques. Cette étude a été réalisée dans le cadre du Master : " Géologie, Océanologie, Eau et SIG», Laboratoire Risques Géologiques, Télédétection et Développement Durable (GEORISK), Faculté des Sciences de Rabat. Nous tenons à remercier les professeurs de ce Master et les évaluateurs pour leurs commentaires et suggestions.

\section{Références}

1. R. Bouatmani, Le bassin d'Essaouira (avantpays atlasique, Maroc) : évolution géodynamique méso-cénozoïque et modélisation de la maturation thermique des hydrocarbures. Thèse Doctorat, Université Mohammed V Agdal, Faculté Sciences Rabat, 206 p. (2002)

2. P. Broughton, A. Trepanier, Hydrocarbon generation in the Essaouira basin of western Morocco. A.A.P.G. Bull., 77, 6, 999-1015. (1993)

3. Bertin, CGG. Sismique réfraction. La sismique réfraction appliquée aux problèmes de la mécanique des roches. Bull. BRGM. Section 2. N4 p 19-29. (1970)

4. A. COCHET \& M. COMBE, Bassin d'EssaouiraChichaoua et zone côtière d'Essaouira. Rapp. Notes Et Mémoires Du Service Géologique. $\mathrm{N}^{\circ}$ 231. Inéd. MTPC/DH/DRE. (1975)

5. F. Coppens, Les corrections statiques de base. Fascicule réf 29870. IFP (France). (1982)

6. M. Djeddi, Carottage sismique et Sismosondage. Extrait de http://djeddimabrouk.fr.gd/. Date d'accès: Juillet 2016 (2013)

7. M. Djeddi, Cours de prospection sismique «Les Corrections Statiques» Département de Géophysique, Université M'Hamed Bougara de Boumerdes. Algérie. Nov. 22 p. (2015)

8. P. Le Roy, Les bassins ouest marocains, leur formation et leur évolution dans le cadre de l'ouverture et du développement de l'Atlantique central (marge africaine). Thèse de Doctorat, Université de Bretagne occidentale, Brest, France, 327 p. (1997)

9. ONAREP, Triassic depositional system: geology and play concept. Second Seminar on Petroleum exploration in Morocco, Rabat, 24-25 november 1998 (inédit), 105 p. (1998) 